定义 $\delta$ 阶 Riesz 平均为:

$$
\begin{aligned}
& S_{R}^{\delta}(f, x)=\left(1-\frac{|\beta|^{2}}{R^{2}}\right)^{-\delta} \sum_{|\lambda+\beta|<R} \\
& \cdot\left(1-\frac{|\lambda+\beta|^{2}}{R^{2}}\right)^{\delta} \operatorname{tr}\left(C_{\lambda} \Phi_{\lambda}^{\prime}(x)\right)
\end{aligned}
$$

这里 $\beta-\frac{1}{2} \sum_{\varepsilon \in \Delta^{+}} \xi, \Phi_{\lambda}(x)=\sqrt{d_{\lambda}} U_{\lambda}(x)$,

$U_{2}$ 是以 $\lambda$ 为最高权的 $G$ 的不可约酉表示，

$$
\begin{gathered}
d_{2}=\prod_{\xi \in \Delta^{+}}(\lambda+\beta, \xi) /(\beta, \xi), \\
C_{2}=\int_{G} f(x) \overline{U_{\lambda}(x)} d x^{[1]} .
\end{gathered}
$$

我们讨论了 Riesz 平均在临界指标 $\alpha_{0}$ $-\frac{\operatorname{dim} G-1}{2}$ 以下逼近函数的性质, 得到如

下的上界估计:

定理 1 设 $\omega(t)$ 是连续模, $H_{\omega}(G)=$ $\{f(x)|| f(x)-f(y) \mid \leqslant \omega(d(x, y))\}$, 定义 量

$$
\begin{aligned}
& \mathscr{\mathscr { S }}\left(S_{R}^{\delta}(f), H_{\omega}(G)\right) \\
& \quad=\sup _{f \in H_{\omega}(G)}\left\|f(x)-S_{R}^{\delta}(f, x)\right\|_{\epsilon}
\end{aligned}
$$

则

$$
\leqslant\left\{\begin{array}{c}
\mathscr{E}\left(S_{R}^{\delta}(f), H_{\omega}(G)\right) \\
A_{1} \omega(1 / R) \ln R+O(\omega(1 / R)) \\
+O\left(R^{-1} \ln R\right), \delta=\alpha_{0} \\
A_{2} R^{\alpha_{0}-\delta} \omega(1 / R)+O\left(R^{\alpha_{0}-\delta-\frac{1}{2}}\right), \\
\frac{l-1}{2}<\delta<\alpha_{0},
\end{array}\right.
$$

其中 $A_{1}, A_{2}$ 是可以算出的常数.

当 $\delta-\alpha_{0}, \omega(t)$ 是凸的, 我们给出了在
环群上尚未知的 Колмогов-Никольский 问题 的解。

定理 2 设 $\omega((t))$ 为任意凸连续模， 则

$$
\begin{aligned}
\mathscr{E}\left(S_{R^{0}}^{a_{0}}(f), H_{\omega}(G)\right)= & C(\omega(2 \pi / R)) \ln R \\
& +O(\omega(1 / R)),
\end{aligned}
$$

其中 $C=\left(\sqrt{\frac{2}{\pi}} \int_{\sigma}|D(\eta)|^{2} d \sigma_{\eta}\right)\left[(2 \pi)^{l / 2}|W|\right.$ $|D(\beta)|]^{-1}, \sigma$ 是 $l$ 维单位球面, $D(h)=\sum_{\ell \in \Delta^{+}}$ $(h, \xi)$.

利用定理 $1 、 2$ 证得如下的逼近定理.

定理 3 设 $W^{k} H^{\infty}(G)=\left\{f(x) \mid f^{(k)}(x)\right.$ $\left.\in H_{\omega}(G)\right\}$ 则

$$
\mathscr{E}\left(S_{R}^{\delta}(f), W^{k} H_{\omega}(G)\right)
$$

$$
=\sup _{f \in W^{k} H_{\sigma}(G)}\left\|S_{R}^{\delta}(f, x)-f(x)\right\|_{c}
$$

$$
\leqslant\left\{\begin{array}{l}
C \omega(1 / R), \delta+K>\alpha_{0} \\
C \omega(1 / R) \ln R+O(\omega(1 / R)) \\
\quad+O\left(R^{-1} \ln R\right), \delta+K=\alpha_{0} \\
C R^{a_{0}-\delta-k} \omega(1 / R)+O\left(R^{\frac{n-3}{2}-\delta-k}\right), \\
\frac{l-1}{2}<\delta+K<\alpha_{0} .
\end{array}\right.
$$

\section{考文献}

[1] Clerc, J. L., Ann. Inst. Fourier Grenoble, 24 (1974), 1: $147-172$.

赵和生

（安微大学数学系,合肥）

\title{
Gronwall-Bihari 积分不等式的某些推广
}

本文旨在给出几种类型的 GronwallBihari 积分不等式解的新的估计式. 以下定 理纠正或推广了 D. Willett, U. D. Dhongade
和张炳根最近的一些结果.

定理 1 设 $f(x) \geqslant 0, g_{i}(x) \geqslant 0$ ， $h_{i}(x) \geqslant 0$ 在 $R_{+}$上连续, 如

科学通报 


$$
y(x) \leqslant f(x)+\sum_{i=1}^{\infty} g_{i}(x) \int_{0}^{x} h_{i}(s) y(s) d s,
$$

则

$$
\begin{gathered}
y(x) \leqslant A_{n}(f)+A_{n}\left(g_{n}\right) \int_{0}^{x} h_{n} A_{n}(f) \\
\exp \left(\int_{s}^{x} h_{n} A_{0}\left(g_{n}\right) d t\right) d s,
\end{gathered}
$$

其中函数 $A_{n}(u)$ 由下式定义:

$$
\begin{aligned}
& A_{1}(u)=u, \\
& A_{k+1}(u)=A_{k}(u)+A_{k}\left(g_{k}\right) \int_{0}^{x} h_{k} A_{k}(u) \\
& \exp \left(\int_{s}^{x} h_{k} A_{k}\left(g_{k}\right) d t\right) d s . \\
& (k=1,2, \cdots, n)
\end{aligned}
$$

定义 称函数 $\omega: R_{+} \rightarrow R_{+}$属于函数 类 $\mathscr{F}$, 如果

a) $\omega(u) \geqslant 0$ 在 $R_{+}$上非减连续,
b) $\frac{1}{v} \omega(u) \leqslant \omega\left(\frac{u}{v}\right)$ 对 $u \geqslant 0, v \geqslant 1$ 成立,

c) $\omega(u)$ 在 $R_{+}$上次可加.

注 如 $\omega(u) \geqslant 0$ 是非减可微凸函 数, 则 $\omega(u) \in \mathscr{F}$.

定理 2 设 $f(x), g_{i}(x), h_{i}(x)(i=$ $1,2, \cdots, n)$ 及 $A_{n+1}(u)$ 如定理 1 所设, $g_{n+1}(x)>0, h_{n+1}(x) \geqslant 0$ 在 $R_{+}$上连续, $\omega(u) \in \mathscr{J}, \phi(u) \geqslant 0$ 在 $R_{+}$上非减连续, 如

$$
\begin{aligned}
y(x) & \leqslant f(x)+\sum_{i=1}^{n} g_{i}(x) \int_{0}^{x} h_{i}(s) y(s) d s \\
& +g_{n+1}(x) \phi\left(\int_{0}^{x} h_{n+1}(s) \omega(y(s)) d s\right),
\end{aligned}
$$

则

$$
\begin{aligned}
& y(x) \leqslant A_{n+1}(f)+A_{n+1}\left(g_{n+1}\right) \\
& \cdot \boldsymbol{\phi}\left(F ^ { - 1 } \left[F \left(\int_{0}^{x} h_{n+1} \bar{A}_{n+1}\left(g_{n+1}\right)\right.\right.\right. \\
& \left.\cdot \omega\left(\frac{A_{n+1}(f)}{A_{n+1}\left(g_{n+1}\right)}\right) d s\right) \\
& \left.\left.+\int_{0}^{x} h_{n+1} \bar{A}_{n+1}\left(g_{n+1}\right) d s\right]\right),
\end{aligned}
$$

$x \in[0, b)$

其中

$$
\begin{gathered}
\bar{A}_{n+1}\left(g_{n+1}\right)=\max \left\{A_{n+1}\left(g_{n+1}\right), 1\right\}, \\
F(u)=\int_{u_{0}}^{u} \frac{d s}{\omega(\psi(s))}, u_{0} \geqslant 0, u \geqslant 0 .
\end{gathered}
$$

定理 3 设 $f(x), g_{i}(x), h_{i}(x)(i=$ $1,2, \cdots, n)$ 及 $A_{n+1}(u)$ 如定理 1 所设, $g_{n+1}(x)>0, h_{n+1}(x) \geqslant 0$ 在 $R_{+}$上连续, 且 $A_{n+1}\left(g_{n+1}\right) \leqslant 1, \omega(u) \geqslant 0$ 是 $R_{+}$上的非减 连续凹函数, $\omega(0)=0, \psi(u) \geqslant 0$ 在 $R_{+}$上 非减连续, 若

$$
\begin{aligned}
y(x) \leqslant & f(x)+\sum_{i=1}^{n} g_{i}(x) \int_{0}^{x} h_{i}(s) y(s) d s \\
& +g_{n+1}(x) \psi\left(\int_{0}^{x} h_{n+1}(s) \omega(y(s)) d s\right),
\end{aligned}
$$

则

$$
\begin{aligned}
& y(x) \leqslant A_{n+1}(f)+A_{n+1}\left(g_{n+1}\right) \\
& \cdot \varphi\left(I^{-1}[I(0)\right.
\end{aligned}
$$

$$
\begin{gathered}
+\alpha \beta \int_{0}^{x} \frac{h_{n+1} A_{n+1}\left(g_{n+1}\right) \omega\left(\frac{A_{n+1}(f)}{\alpha A_{n+1}\left(g_{n+1}\right)}\right)}{\omega\left(r_{n+1}(s)\right)} d s \\
\left.\left.+\beta \int_{0}^{x} h_{n+1} A_{n+1}\left(g_{n+1}\right) d s\right]\right) . x \in(0, b)
\end{gathered}
$$

对 $\alpha>0, \beta>0, \alpha+\beta=1$ 成立. 其中

$$
\begin{gathered}
r_{n+1}(s)=\int_{0}^{s} h_{n+1} A_{n+1}\left(g_{n+1}\right) \\
\cdot \omega\left(\frac{A_{n+1}(f)}{A_{n+1}\left(g_{n+1}\right)}\right) d t, \\
I(u)=\int_{\alpha_{0}}^{n} \frac{d s}{\omega(\psi(s) / \beta)}, u_{0} \geqslant 0, u \geqslant 0 .
\end{gathered}
$$

在定理 $2 、 3$ 中, $b$ 的决定应使 $F^{-1}$ 或 $l^{-1}$ 有定义。

将上述定理应用于 Volterra 积分方程和 微分方程中, 我们得到一些新的较好的结果。

\section{孔庆凯张炳根}

（山东海洋学院，青岕） 